\title{
Hawking responde a las grandes preguntas. La filosofía de Stephen Hawking. Un comentario sencillo al libro "Breves respuestas a las grandes preguntas".
}

\author{
Hawking answers great questions \\ the Philosophy of Stephen Hawking. \\ A simple remark on the book \\ "Brief Answers to the Big Questions" \\ DOI: https://doi.org/10.5377/koot.v0i11.10732 \\ URI: http://hdl.handle.net/11298/1188
}

Eduardo Badía-Serra Director de la Academia Salvadoreña de la Lengua Miembro del Senado Consultivo de la Universidad Tecnológica de El Salvador ebbserra@yahoo.com.mx Fecha de aceptación: diciembre 2020

\section{Resumen}

El autor analiza las respuestas que Stephen Hawking da a lo que él llama "grandes preguntas", en su libro póstumo "breves respuestas a las grades preguntas". El enfoque es de un grave talante filosófico, al margen de su expresión científica, en virtud del tono con que el gran científico británico atiende tales cuestiones. Es, puede decirse, un análisis del pensamiento filosófico de Hawking, que, por supuesto, este trata de ubicar dentro de las más cerradas aristas de las ciencias. Siempre, él creyó que la ciencia juega un papel definitivo en la resolución de los problemas del hombre y del universo, e incluso afirma que solo las ciencias pudrían dar tales respuestas. En el libro, las intenta.

Palabras clave: Astronomía - Preguntas y respuestas. Cosmología - Preguntas y respuestas. Espacio y tiempo - Preguntas y respuestas. Ciencia - Preguntas y respuestas. Existencia de Dios - Preguntas y respuestas. Metafísica - Preguntas y respuestas. Hawking, Stephen - Pensamiento filosófico. 


\begin{abstract}
Abstrac
The author analyzes the answers that Stephen Hawking gives to what he calls "big questions" in his posthumous book "Brief Answers to the Big Questions". The approach given in this book holds a great philosophical temper--at the margin of his scientific expression-- in honor to the tone with which the great British scientist takes care of such matters. We could say that it is an analysis of Hawking's philosophical thought, which, of course, he tries to place within the sharpest edges of science. He always thought that science plays a definite role in the resolution of the problems faced by humans and the universe; he even affirms that science is the only one who could provide said answers. In this book, he tries to answer them.
\end{abstract}

Key words: Astronomy - Questions and answers. Cosmology - Questions and answers. Space and time - Questions and answers. Science - Questions and answers. Existence of God - Questions and answers. Metaphysics - Questions and answers. Hawking, Stephen - Philosophical thought.

\title{
LOS MENSAJES FINALES DE HAWKING.
}

"Las grandes preguntas de la existencia siguen sin respuesta:

¿Cómo comenzó la vida en la Tierra?

¿Qué es la conciencia?

¿Hay alguien por ahí o estamos solos en el universo?

¿Qué es lo que hace que el universo exista?”

"Nuestro mundo cambiará

enormemente en los próximos

cincuenta años: Descubriremos qué

sucedió en el big bang.

Descubriremos cómo comenzó la vida en la

Tierra. Podremos descubrir si hay vida en otros lugares del universo.

Crearemos hábitats viables para la especie humana en otros planetas.”

"Soy optimista.

Miremos a las estrellas y no a los pies.

Seamos curiosos" 


\section{Introducción}

En respuesta a muchas preguntas que se le hacían regularmente, Stephen Hawking, abriendo los contenidos de su archivo personal, nos las revela ahora en una publicación póstuma, un excelente libro, "Breves respuestas a las grandes preguntas". El gran científico y filósofo británico responde efectivamente a grandes preguntas: ¿Hay un Dios?, ¿Podemos predecir el futuro?, ¿Qué hay dentro de un agujero negro?, ¿Nos superará la inteligencia artificial?, ¿Hay más vida inteligente en el universo?, ¿Deberíamos colonizar el espacio?, ¿Cómo damos forma al futuro?, ¿Es posible viajar en el tiempo?, ¿Sobreviviremos en la Tierra?, ¿Cómo empezó todo? Enfoca cada una de ellas separadamente, pero en su conjunto, se puede ver sin dificultad que tiene él una posición unificada en un todo que podría resumirse en: ¿Qué hace el hombre en la Tierra?, ¿Cuál es su papel?, ¿Cómo debe reaccionar ante el futuro? En el fondo, una sola pregunta, una sola preocupación. Hawking trata de responder bajo el manto de la ciencia, que siempre le ha sabido cobijar, pero no puede marginarse que hay en él una búsqueda de carácter filosófico y teológico incluso, por más que siempre haya tratado de expresar su rechazo, aunque moderado, a estas dos formas de conocer.

Su amigo y colega Kip S. Thorne, que prologa el libro, expresa al final de este que Hawking siempre se sintió atraído por las grandes preguntas, al margen de si eran cosa de la ciencia o no. Y puntualiza muy sintetizadamente las respuestas que él da, respuestas estas que intentaré detallar un poco más en las páginas que siguen, procurando respetar las propias expresiones del autor y de interpretar lo mejor posible sus contenidos y el sentido que él quiere darle.

En primer lugar, su afirmación y muy defendida posición de que el universo tuvo un comienzo, que debió haber comenzado en un tiempo finito, con lo cual no puede ser infinitamente viejo. Es en las singularidades donde comenzó el espacio-tiempo, dice. Aquí confronta con Einstein, aunque al final, pareciera haber un acuerdo, más porque este último modificara su posición inicial de un universo estable e invariable, que porque Hawking cambiara la suya. Recordemos que ambos genios nunca se conocieron personalmente, pues Einstein murió cuando Hawking era solo un niño. En opinión de Hawking, el universo debió haber comenzado en algún tipo de estado singular, hace aproximadamente unos 10,000 millones de años. Sus trabajos con su amigo y colega Roger Penrose concluyeron en que en el centro de un agujero negro se aloja una singularidad en la que el tiempo termina, aunque, como ya he citado, es en las singularidades en donde también comenzó el espacio-tiempo. Es importante reseñar esto porque debemos recordar que la ciencia reconoce que, para las singularidades, ella no tiene explicación válida. Esta es una limitación que siempre encuentro en las posiciones de Hawking cuando trata de sostener que sólo la ciencia es capaz de explicarse las grandes preguntas, a pesar de que concluye siempre también de que hoy por hoy dichas explicaciones fallan. 
Hay un enfoque también muy pronunciado en sostener sus dos famosas teorías de la "temperatura de Hawking" y de la "radiación de Hawking", verdaderamente radicales, y que, dice Thorne, "abrieron los ojos a conexiones profundas entre la realidad general, (agujeros negros), la termodinámica, (la física del calor), y la física cuántica, (la creación de partículas donde antes no había ninguna). Con ello, Hawking llegó a su demostración de que en los agujeros negros hay entropía, su famosa "entropía residual", con lo cual estos son seres aleatorios, aleatoriedad que debe ser proporcional al área de la superficie del agujero. Cita Thorne que, en la lápida mortuoria de Hawking en la Abadía de Westminster en Londres, donde yacen sus cenizas entre las de Isaac Newton y Charles Darwin, está grabada la fórmula para calcular la "temperatura de Hawking", y que pronto, la fórmula para calcular la entropía residual de un agujero negro será grabada también en la piedra memorial de Hawking en el College Gonville and Caius Cambridge, Inglaterra, donde trabajaba. Los científicos son muy dados en grabar esos recuerdos en lápidas y otros monumentos: Solamente deseo recordar aquí que en la lápida mortuoria de Werner Heisenberg se ha grabado una frase que refleja su famoso principio de incertidumbre, que más o menos dice que allí reposan, "en algún lado", no en uno preciso, los restos del gran científico alemán; y también, que en la lápida de otro gran científico germano, Ludwig Boltzmann, se dejó grabada su fórmula inicial para el cálculo de la entropía absoluta, $\mathrm{S}$ $=\mathrm{k} \log \mathrm{W}$, transformada luego a la forma $\mathrm{S}=\mathrm{k} \ln \mathrm{W}$. Hawking, sobre la base, aunque no sólo de sus estudios sobre los agujeros negros, siempre razonó que si se llegaba a un punto de unión y complementación entre la física cuántica y la relatividad general, debía dar como resultado la destrucción de la información. -Pienso yo - , detendría el avance del universo. Einstein también sostuvo una posición similar, aunque expresada en términos diferentes. Decía él que "algo faltaba en la física cuántica" que la hacía científicamente incompleta, lo que le llevó a las profundas y ahora muy convenientes discusiones con su gran amigo y colega Bohr. A pesar de ello, Hawking sostiene al final, matizando su posición, que "la información no se pierde, pero no se recupera de manera útil". Es, dice, "como quemar una enciclopedia, pero reteniendo el humo y las cenizas".

La preocupación de Stephen Hawking sobre las grandes preguntas fue siempre permanente y manifiesta. Él mismo afirma que la ciencia se encuentra cerca de algunas de las respuestas, pero todavía estas respuestas no se tienen. En algunas ocasiones, afirma que la filosofía y la teología no sólo no son referentes adecuados y oportunos al caso, sino que más bien estos enfoques son limitados y hasta perjudiciales. En "Agujeros negros y pequeños universos", (Tribuna, Plaza y Janes Editores, 1993, Barcelona) ya nos lo va diciendo. Los científicos son muy dados a reconocer sus errores, y a cambiar sus posiciones, con lo cual revelan y confirman el carácter no dogmático de la ciencia. En esta oportunidad, Hawking nos va diciendo, (VI. Mi posición), que “Quienes deberían estudiar y 
debatir tales cuestiones, los filósofos, carecen en su mayor parte de preparación matemática suficiente para estar al tanto de las últimas evoluciones registradas en la física teórica...", y es hasta cáustico cuando continúa a renglón seguido que “...Existe una subespecie, la de los llamados filósofos de la ciencia, que tendría que hallarse mejor equipada al respecto...". Ahora, en este nuevo libro póstumo, ha dejado dicho que "...Mientras haya vida, hay esperanza", esperanza que, sin temor a equivocarme, él la cifra en la ciencia, aunque en un lugar muy recóndito de su mente acepte que alguna probabilidad existe de que no sea la ciencia sino la filosofía y la religión quienes puedan llegar a una respuesta, aunque sea sólo más cercana. Hawking es, sin duda alguna, un hombre de fe, pero un hombre de una fe no convencional, sino una fe en un ser superior, no personal. Einstein, de nuevo, participaba de una fe similar; baste recordar su pensamiento cuando hablaba del eterno retorno y del sentimiento cósmico religioso.

Hawking fue siempre un hombre anclado en la realidad, pero profundamente influido por la ciencia. Ese fue su carácter definitivo. La ciencia no le dejó respirar otros ambientes, al menos de manera tan profunda y seria como lo hizo en aquella. La ciencia le hizo negar, diciéndolo de alguna manera, la religión, el mito, la tradición, la filosofía. Muy a pesar de ello y de una mente centrada en la ciencia, comprendió que el hombre, al margen de que debía buscar afanosa y necesariamente una respuesta a esas grandes preguntas que ya hemosseñalado, también tenía otras preocupaciones, otros grandes desafíos, ¿cómo alimentar a una población en creciente crecimiento, proporcionar agua limpia, generar energía renovable, prevenir y curar enfermedades, y frenar el cambio climático global? Aquí en esto último hago alguna consideración. Habla él de un "cambio climático global", de origen natural, propio de la dinámica universal misma, y no de un cambio climático local, y menos aún producido por el hombre, presionado este siempre por la creencia en su antropocentrismo radical y fatal, y en su creencia de que es el hombre quien debe dictarle a la naturaleza cómo debe conducirse, y no al revés.

\section{1}

\section{¿Hay un Dios?}

Resumamos: Según Hawking (2018) “¿Cómo encaja la existencia de Dios en su comprensión del inicio y del final del universo? Y si Dios existiera y usted tuviera la oportunidad de encontrarse con él, ¿qué le preguntaría?" (p. 52). Así orienta Hawking las preguntas sobre si hay un Dios, y las responde: Cree que el cómo empezó el universo no fue una cosa escogida por Dios por razones que no podemos comprender, sino que fue determinada por una ley de la ciencia. Él acepta que pueda llamarse Dios a las leyes de la ciencia, pero no sería un dios personal al que pudiera encontrarse y preguntarse tales cosas. 
Respuesta si se quiere elusiva, a lo lejos, metafísica, de una metafísica ligera e insustancial, si es que, como veremos más tarde, las leyes actuales de la ciencia no responden a la pregunta por los primeros instantes, y parece que no hay más acercamientos a ello, superando los tiempos, los espacios y las masas de Planck determinadas con el uso de sus "Constantes Naturales". (Hawking, 2018, p. 52). Pero veamos cómo fundamenta Hawking sus respuestas a la pregunta de ¿Hay un Dios? Primero aclara que no tiene ningún resentimiento hacia Dios, ni quiere dar la impresión de que él ha tratado de refutar su existencia. Esto es muy importante de comprender. Es un hombre de fe, indudablemente, pero su fe la expresa de manera diferente, es una especie de "sentimiento cósmico religioso", al decir de Einstein, que delata su profunda confrontación interna ante esta incógnita universal e infinita. Claro que objeta: Si se acepta, como él lo hace, que las leyes de la naturaleza son fijas, ¿Qué papel queda para Dios? Aquí expresa un determinismo total, que luego confronta cuando hace entrar la indeterminación introducida por Heisenberg. Conocer la mente de Dios es conocer las leyes de la naturaleza. Cree, tajantemente, que para finales del siglo actual, el hombre, a través de la ciencia, conocerá la mente de Dios, pero luego entra en la contradicción: ¿Será la existencia de Dios una pregunta válida para la ciencia?, a pesar de que insiste en afirmar que el universo fue creado espontáneamente de la nada, según esas leyes, que "pueden, o no, haber sido decretadas por Dios, pero este no puede intervenir para transgredirlas, o no serían leyes", con lo cual deja a Dios la libertad de elegir el estado inicial del universo, pero incluso aquí, como habría leyes, Dios no tendría ninguna libertad. Yo siento que, Hawking sostiene que Dios es la única forma de abordar el origen del universo, y que en este abordaje, el papel desempeñado por el tiempo es la clave definitiva para eliminar la necesidad de un gran diseñador y para revelar cómo el universo se creó a sí mismo. El origen finito del tiempo es, pareciera, para Hawking (2018), la solución a la contradicción, y lo remarca: "Cuando la gente me pregunta si Dios ha creado el universo, les digo que la pregunta no tiene sentido. Antes del big bang el tiempo no existía, y por lo tanto no había un tiempo en que Dios pudiera hacer el universo" (p. 52). Bien, pero ¿es que el "todo" sólo está constituido por el universo? ¿Quién puede afirmar que fuera del universo no exista algo, superior infinitamente? Y, además, a pesar de la profunda, científicamente demostrada por él, teoría de que el tiempo tuvo un comienzo, este es ahora un asunto no aceptado y más bien debatido por la comunidad científica.

Hawking "tiene fe", él mismo lo afirma y lo acepta. Y su fe la lleva a aceptar que no hay Dios. Así lo expresa. "No hay cielo ni vida futura”. ¡Terrible afirmación! 


\section{Pero, entonces, ¿Cómo empezó todo?}

Yo siempre, en los escritos de Hawking, me he preguntado por qué él usa el término Dios tanto en minúsculas como en mayúsculas; y no creo que sea razonable pensar que ha sido por mera distracción o por que ello sea de poca importancia. Más bien pienso que en él, como en todos los hombres y probablemente más en él, que fue una mente inquieta como la de todo científico, esto le oprimía. Pienso que nunca pudo liberarse de tal opresión.

Igual que Penrose, sostenía que el universo "debió" haber tenido un comienzo. Matemáticamente, esto pareciera irrefutable, y ello no se opone a la visión teológica y filosófica. El asunto es que dicho comienzo parecería no explicable por la ciencia. El mito y la religión sí lo explican muy bien. Para la ciencia, el universo comenzó con el big bang, término despectivo en su origen, y ello dejaba sin efecto la relatividad general, restándole validez, que, como es aceptada y demostrada científicamente, dejaba fuera del alcance de la ciencia tal explicación.

Aquí recurre Hawking al principio antrópico, que en su forma dura, como sabemos, afirma que el universo tiene que ser más o menos como lo vemos porque de otra manera no habría nadie para observarlo. El concepto de tiempo sólo existe en el universo, y en el hombre, agregaría yo. ¿Dios juega a los dados, o es un jugador empedernido? (Hawking, 2018, p. 68)

¿Y el hombre?

Siempre pensó Hawking que el hombre, como forma de vida inteligente, y pudiendo dominar la técnica de los viajes espaciales, debería prepararse para trasladarse a otra estrella. De otra forma, la vida en la tierra estaría condenada al fracaso. La vida en la tierra comenzó hace unos 500 millones de años, por una especie de generación espontánea. La evolución humana, por su lado, comenzó en su etapa crítica, con el desarrollo del ácido desoxirribonucleico (ADN), el desarrollo del lenguaje, y en lo particular, el lenguaje escrito, que significa que la información puede transmitirse de generación en generación no sólo por medio del ADN. Dice Hawking, y esto es muy relevante para nuestra comprensión de nosotros mismos, que: "La cantidad total de información útil en nuestros genes es probablemente algo así como cien millones de bits, siendo un bit, la información que posibilita una respuesta del tipo sí o no" (Hawking, 2018, p.87). Calculando un poco, concluye que "un ser humano es equivalente a unos cincuenta libros de Harry Potter, y una gran biblioteca nacional puede contener alrededor de cinco millones de libros, esto es, más o menos, 10,000 millones de bits. La cantidad de información transmitida en libros o por internet 
es unas 100,000 veces mayor que en el ADN". Y ello le lleva a considerar que la información útil del hombre en su ADN probablemente ha cambiado en sólo unos pocos millones de bits, con lo que la evolución humana de tipo biológica es de sólo un bit por año. Ello, acoto yo, debiera llevar a la reflexión de aquellos que pretenden que el hombre pueda frenar el cambio climático global.

Hawking cree a pie tendido en la posibilidad, y más bien, en la necesidad, de los "superhumanos". Si el hombre no se vuelve un "superhumano", y permanece como humano no mejorado, no podrá competir con ellos, morirá, o en el mejor de los casos perderá importancia. Hay pues, que "rediseñar a la especia humana" para reducir el riesgo de su destrucción o de su autodestrucción. Esta será evitable haciendo del hombre un ser inmigrante, no por supuesto al sueño americano, acoto, sino más bien emigrar y colonizar otras estrellas o planetas, eso sí, estrellas o planetas cercanas para no hacer imposible para nuestras condiciones el tiempo del viaje. Hawking cree en la existencia de muchas otras estrellas cuyos planetas alberguen vida. Y la Tierra no ha sido "visitada por otros "seres", (los ovnis no contienen seres del espacio exterior), porque de ser así, la visita de extraterrestres hubiera sido "mucho más manifiesta y probablemente más desagradable".

Concluye Hawking (2018) diciendo que "un encuentro con una civilización más avanzada, en nuestra etapa actual, podría resultar un poco como cuando los habitantes originales de América conocieron a Colón” (p. 95), y no cree él que mejorarían con tal encuentro.

\section{3}

\section{La "teoría de variables ocultas" y la predicción del futuro.}

La visión de Laplace del determinismo científico fue socavada por el indeterminismo del principio de incertidumbre de Heisenberg, de acuerdo con la cual no sería posible predecir el futuro. ¿Cómo puede predecirse el futuro si ni siquiera se pueden conocer con suficiente exactitud la posición y la velocidad de una partícula? Esto, por cierto, es una crítica al absolutismo de las computadoras, que, por muy poderosas que estas sean, si se le introducen datos deficientes producirán predicciones deficientes. Como hemos dicho ya, Einstein no aceptaba que la naturaleza actuara aleatoriamente, a pesar de sus dudas internas en cuanto al asunto. Cuando dijo "Dios no juega a los dados", se siente que lo dijo dudando de ello. Él creía que la incertidumbre era una cuestión provisional, y que en el fondo la naturaleza era determinista y en ella todo estaba definido.

Esta visión de Einstein es lo que se conoce ahora como teoría de variables ocultas, con lo cual trataba de conciliar incorporando el principio de 
incertidumbre a su posición determinista. Hawking pensaba que esta teoría era incorrecta, como parece haber comprobado el físico británico John Bell. Según esta comprobación, Dios está sujeto también al principio de incertidumbre. Dios juega a los dados con el universo, y Hawking siempre remarcó ello diciendo que no sólo jugaba a los dados, sino que era un jugador inveterado. Esto es importante de considerar, aunque sin embargo el que Dios juegue a los dados con el universo no significa, al menos necesariamente, que no haya sido Él quien lo creó.

Hawking, ante la pregunta de si las leyes que rigen el universo permiten predecir exactamente lo que va a suceder en el futuro, responde que "la respuesta breve es no, y sí", y la dificultad la hace residir, no en las leyes de la ciencia sino en la dificultad de los cálculos. (Hawking, 2018, p. 101)

\section{4}

\section{¿Es posible viajar en el tiempo?}

¿Tiene algún sentido organizar una fiesta para viajeros en el tiempo? ¿Esperaría que alguien regresara del futuro? Así se pregunta Hawking, resumiendo la respuesta a este enigma natural muy coloquialmente:

"En 2009 organicé una fiesta para viajeros del tiempo en mi College, Conville y Caius en Cambridge, para una película sobre viajes en el tiempo. Para asegurarme de que sólo llegaran viajeros genuinos en el tiempo, no envié las invitaciones hasta después de la fiesta. El día señalado, me senté en la universidad esperando, pero nadie vino. Me decepcionó, pero no me sorprendió, porque había demostrado que si la relatividad general es correcta y la densidad de energía es positiva, el viaje en el tiempo no es posible. Me hubiera encantado que alguna de mis suposiciones hubiera sido falsa". (Hawking, 2018, p. 142)

Veamos: Lo que Hawking comprueba con su fiesta, esto es, no puede viajarse en el tiempo, luego lo matiza. No puede ello ser posible, aunque no de manera absoluta. Su conclusión en este caso es que "los viajes espaciales rápidos o los viajes en el tiempo no pueden ser descartados por nuestra comprensión actual, aunque causarían grandes problemas lógicos, así que es esperable que haya una ley de protección cronológica que evite que 'las personas regresen y maten a nuestros padres"” (Hawking, 2018, p. 143). Esto tiene su explicación.

Ha habido muchos intentos para demostrar que es posible viajar en el tiempo. Hawking refiere algunos de ellos: Kurt Godel, el matemático, encontró en 1948 una solución a las ecuaciones de campo de la relatividad general que representa un universo en el que todo su conjunto está girando, con lo cual, en un universo 


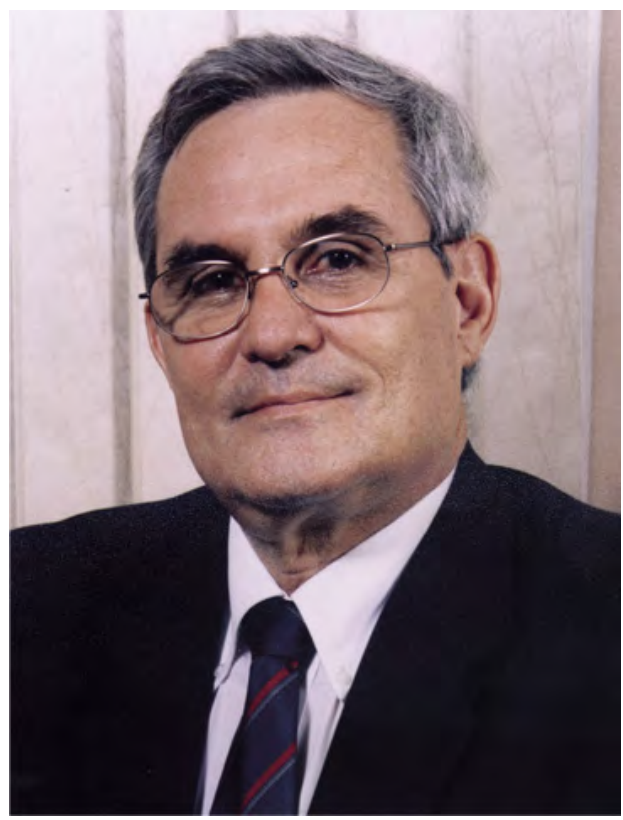

Eduardo Badia Serra

así, una nave espacial podía partir y regresar antes de haber partido. Esto molestó a Einstein, quien no aceptó que su relatividad general pudiera demostrar tal cosa, y aparentemente, el error de Godel fue el no considerar que el universo se expande. Otro intento de demostrar la posibilidad de viajar en el tiempo es el de las llamadas cuerdas cósmicas viajando una respecto a la otra casi a la velocidad de la luz. Un tercer intento, expone Hawking, es el del llamado Efecto Casimir, muy conocido. Un cuarto intento que él describe es el de la teoría de la "Suma de Historias", expuesta por el físico Richard Feynman:

Según la teoría cuántica, el universo no tiene una única historia sino todas las historias posibles, cada una de ellas con su propia probabilidad; pero en algunas historias, el espacio-tiempo estará tan curvado que los objetos como los cohetes podrán viajar al pasado. Esto es lo que precisamente Hawking intentaba ver en su fiesta. Pero resulta que cada historia es completa y autónoma, y describe no sólo el espacio-tiempo curvo sino todos los objetos que contiene, por lo que un cohete no puede transferirse a otra historia alternativa cuando regresa de nuevo, es decir, todavía se encuentra en la misma historia, que tiene que ser autoconsistente. ¿A qué lleva lo anterior? A demostrar que si elespaciotiempo está tan deformado que las posibilidades de viajar en el tiempo son realmente muy pequeñas, reducidas a una región microscópica. 
Hawking comienza su argumentación exponiendo, a manera de ilustración, las características de las diferentes geometrías no euclidianas, particularmente las de Riemann y Lovachevski, así como la conexión existente entre el espacio y el tiempo en el espacio-tiempo. Ello para poder demostrar posteriormente que todo lo que se necesita para viajar en el tiempo es una nave espacial que pueda viajar a una velocidad mayor que la velocidad de la luz; pero ya Einstein había predicho que para ello se necesitaría una potencia infinita. La evidencia, ahora ya irrefutable, de que el espacio y el tiempo están deformados, y la imposibilidad de viajar a una velocidad mayor que la de la luz, hacen que la posibilidad de viajar en el tiempo regresando al pasado sea infinitesimalmente muy pequeña. No pueden descartarse tales posibilidades, y Hawking señala que la posibilidad real para que ello suceda es que se compruebe experimentalmente la teoría $\mathrm{M}$, esto es, una teoría que unifique la teoría de cuerdas, y que dé la esperanza de unir la relatividad general con la teoría cuántica en una "Teoría del Todo". En ella, el espacio-tiempo debería tener once dimensiones y no sólo las cuatro que experimentamos; de estas once dimensiones, siete, dice Hawking (2018), "están acurrucadas en un espacio tan pequeño que no las notamos", (p. 142) y sólo los cuatro restantes, las que conocemos, son bastante planas y constituirían lo que se llama espacio-tiempo. Pero si se lograra que las cuatro dimensiones planas conocidas del espacio-tiempo se mezclaran con las siete desconocidas altamente curvadas o deformadas, la posibilidad de viajar en el tiempo sería entonces mayor.

\section{5}

\section{¿Sobrevivir en la Tierra o colonizar el espacio?}

En mi opinión, Hawking pareciera señalar que el hombre está siendo sujeto de tres ataques que lo obligarán, para él sin ninguna duda, a escapar de la Tierra colonizando el espacio. No hay posibilidad alguna de que el hombre pueda sobrevivir en la Tierra, en los términos de un mediano plazo.

Primero, un ataque de la naturaleza: Hawking reconoce el cambio climático y el calentamiento global, lo hace dramáticamente y señala al hombre como su causante, pero cree que ambos fenómenos pueden revertirse con la tecnología de la que ya se dispone al momento, y que lo que hace falta es voluntad política. El hombre, dice, se está quedando sin espacio, y los únicos que le estarán disponibles se encuentran en otros mundos.

Segundo, un ataque del hombre mismo contra sí mismo: La Tierra puede ser destruida por un ataque nuclear, por efecto de una guerra nuclear. El hombre se está acercando progresivamente a esta posibilidad, como producto de la ceguera y la necedad de los principales dirigentes políticos. 
Y finalmente, un ataque espacial: Hawking cree que el universo no es un lugar muy amigable, y menos, atractivo; antes bien, es un lugar violento, poblado por seres violentos, como las supernovas, los agujeros negros, los asteroides, etc. Estos seres podrían desencadenar procesos que podrían terminar con la vida en la Tierra.

Hay, pues, para él, "razones por las cuales deberíamos aventurarnos en el espacio, en lugar de quedarnos quietos" (Hawking, 2018, p. 148). Como el hombre es un explorador natural, cosa que le eleva espiritualmente y le ennoblece, así dice, que este salga de la Tierra es una necesidad y un deber, visto esto bajo una escala de globalidad. Explorar otros sistemas solares puede ser la única salvación. Si esto no se hace, la catástrofe es inminente, e incluso la sitúa muy tajantemente en un año, el año 2600. Para ese año, dice, "toda la población estaría en pie, hombro contra hombro, y el consumo de electricidad haría que la Tierra brillara al rojo vivo. Si fuéramos poniendo uno al lado de otro los nuevos libros que van siendo publicados tendríamos que movernos a unos $60 \mathrm{~km} / \mathrm{h}$ para mantener el ritmo con el extremo de la línea. Por supuesto, hacia 2600, los nuevos trabajos artísticos y científicos vendrán en formatos electrónicos en lugar de libros y documentos físicos. Sin embargo, si continuara ese crecimiento exponencial, aparecerían diez nuevos artículos por segundo en mi especialidad de física teórica y no habría tiempo para leerlos" (Hawking, 2018, p. 152).

¿Cuál es la alternativa? Y aquí vuelve a remarcar en la necesidad de unir la relatividad general con la teoría cuántica en una teoría única del Todo", la única forma que el hombre tendría para poder dominar la naturaleza. Ello, unido a que la tecnología permita que pueda aumentarse la complejidad del ADN humano de tal forma que no sea necesario esperar el lento proceso de la evolución biológica, llevando a un rediseño total y completo del hombre en los próximos mil años. En síntesis, Hawking apunta aquí al diseño de humanos mejorados altamente, con todo y los problemas sociales y políticos que ello acarrearía en relación con los humanos que permanecieran no mejorados. En el fondo, que los sistemas electrónicos puedan avanzar y situarse delante de los sistemas biológicos, o al menos al mismo nivel. Hawking dice que eso le preocupa y no lo desea, pero debe aceptarlo porque será inevitable, no en los próximos cien años, pero sí antes que finalice el milenio.

¡Hay que prepararnos para el espacio! Esto lo viene señalando ya desde principios del siglo. Hablaba entonces de la "civilización poshumana", del hombre geneticum y del "Armagedón tecnológico". Ahora, en sus breves respuestas a las grandes preguntas, lo confirma y vuelve sobre ello. ¡Hay que colonizar el espacio!, dice, y ello, para él, no será caro en relación con otros gastos que el hombre hace sobre asuntos menos sustantivos. 
Primero, dice, la Luna; luego, Marte. Esos son los primeros pensamientos. Pero Hawking no confía mucho en ellos como un lugar para vivir sino más bien como una base para viajar luego al sistema solar. A Marte, incluso, lo siente frágil y además la llegada del hombre podría contaminarlo o contaminarse él mismo con su contacto, contaminando a la Tierra si hay un retorno. Más bien, piensa él en Júpiter y Saturno, en las lunas de estos planetas; y, saliendo ya del sistema solar, en aquellos espacios que se estima que son parecidos a la Tierra, y que están en la forma de ser habitados. Propone diez, de los cuales habla de "Próxima b".

Reconoce Hawking que estas son posibilidades inmensamente remotas, pero que no son asunto de ciencia ficción sino de ciencia real. Está seguro de que deben buscarse y encontrarse opciones. Y de nuevo, ello lo ve en la unión de la relatividad general con la teoría cuántica, como ya se ha dicho, así como en el desarrollo del hombre modificado, del "hombre poshumano", del "hombre geneticum".

Hawking pareciera fantástico en cuanto a este tema. Pero él es Stephen Hawking, y sus repuestas vienen de un genio, con lo cual de ninguna manera podrían desestimarse. Es probableque tengarazón, y que sean las limitaciones expectativas del hombre actual las que no permitan leer el futuro tal y como él lo hace.

\section{6}

¿Nos sobrepasará la inteligencia artificial?

Este pareciera ser un asunto de gran debate mundial en los círculos científicos y tecnológicos, probablemente debido al avance extraordinario de los sistemas computacionales y de otros tipos. Hawking es consciente de que la inteligencia artificial ayudaría a la humanidad a resolver muchos de los graves problemas que la aquejan, particularmente en áreas tan sensibles como la salud, la alimentación, los procesos productivos, etc.; pero también lo es en el sentido de los graves problemas que podría ocasionar su mal uso, uno de estos, por ejemplo, que señala repetidamente, la inadecuada manipulación del ADN. Sin embargo, aconseja que el hombre no tema al cambio sino haga que este funcione a su favor. "Cuando inventamos el fuego - dice -nos equivocamos repetidamente y luego inventamos el extintor. Pero con tecnologías más poderosas como por ejemplo armas nucleares, biología sintética e inteligencia artificial fuerte, deberíamos planificar el futuro y tratar de hacer las cosas bien a la primera, porque puede ser la única oportunidad que tengamos. Nuestro futuro es una carrera entre el poder creciente de nuestra tecnología y la sabiduría con que la usemos. Asegurémonos de que gane la sabiduría". Aquí, sólo acoto: El hombre produce la tecnología, en 
este caso, la inteligencia artificial, y la usa; está en el hombre, entonces, definir hasta cuándo y cómo.

Hawking insiste en su concepto del poco desarrollo del cerebro humano, cosa que viene siendo señalada por él en muchos de sus escritos. Para él, no hay diferencia significativa entre cómo funciona el cerebro de una lombriz y la computadora; y tampoco, hay mayor diferencia entre el cerebro humano y el de una lombriz de tierra. Concluye:" Por lo tanto, las computadoras pueden, en principio, emular la inteligencia humana o incluso superarla". Utiliza el genio inglés un silogismo extremadamente clásico en mi concepto, cuando hace tal comparación y deriva tal conclusión. Y continúa comparando, sobre la base de lo anterior: "Cuando una inteligencia artificial supere a los humanos en el diseño de más inteligencia artificial, de modo que pueda mejorarse recursivamente a sí misma sin ayuda humana, podemos enfrentarnos a una explosión de inteligencia que finalmente de lugar a máquinas cuya inteligencia supere a la nuestra en más de lo que la nuestra supera a la de los caracoles". Aquí, el asunto es que la computadora pueda mejorarse sin ayuda humana, recursivamente dice Hawking; pero la mayoría de autores, no todos ciertamente, consideran que esto no será posible, y que las máquinas seguirán dependiendo del hombre. Pareciera que a Hawking le preocupa que la inteligencia superior pueda superar a la inteligencia humana, produciendo máquinas "altamente inteligentes como la ciencia ficción", pero observa y anticipa que descartar tal posibilidad sería un error, y, dice, "potencialmente nuestro peor error". Ello suena un tanto determinista, y fatalista si se quiere.

En este caso de la inteligencia artificial, Hawking, repetidamente, mantiene dudas ocultas pero reales. No puede negarse que, como científico y admirador de la ciencia que es, simpatiza con la idea de que esta pueda superar al hombre e incluso dominarlo, una de las opciones, la inteligencia artificial; pero advierte repetidamente sobre tantos peligros, a la vez que revierte señalando por otro lado tantas ventajas para la humanidad. "El éxito en la creación de inteligencia artificial - dice - sería el mayor acontecimiento en la historia de la humanidad. (Hawking, 2018, p. 176)

"Pasamos mucho tiempo estudiando historia que, seamos sinceros, es sobre todo la historia de la estupidez. Así pues, es un cambio bienvenido que la gente esté estudiando, en lugar de eso, el futuro de la inteligencia" (Hawking, 2018, p. 183); pero casi a continuación, previene: "Por desgracia, (este éxito, este acontecimiento), podría ser el último, a menos que aprendamos como conjurar sus riesgos......Mientras que las formas primitivas de inteligencia artificial desarrolladas hasta ahora han demostrado ser muy útiles, temo las consecuencias de crear algo que pueda igualar o superar a los humanos". 
Entre sus entusiasmos y sus sobresaltos sobre esto de la inteligencia artificial, no cabe duda de que él, el científico, se entusiasma con tal desarrollo, pero también él, el filósofo, se preocupa por sus efectos. De que la inteligencia artificial superará la inteligencia humana, parece, sin embargo, y al margen de lo anterior, que Hawking no tiene duda, a pesar de que siempre también afirmaba que la imaginación era el atributo más poderoso del hombre, y de que la mente humana era algo increíble, y más aún si sabe acompañarse de la investigación y el asombro.

\section{7}

\section{Algunas impresiones finales}

Según Hawking (2018) los restos de Stephen Hawking se encuentran enterrados en la abadía de Westminster, entre los de Isaac Newton y Charles Darwin. Su voz, en el momento de su entierro, fue transmitida telescópicamente por un radiotelescopio hacia un agujero negro. Semejante homenaje, semejante reconocimiento, es, justamente, único, pero justo.

Stephen Hawking fue un eterno esclavo de la ciencia. Creyó en ella ciegamente. Creyó que la ciencia lograría hacer que el hombre superara y resolviera todos sus problemas. También, que la ciencia respondería a las grandes preguntas. Todo lo supeditó a la ciencia.

A pesar de ello, al momento de su muerte, los grandes problemas del hombre no han sido resueltos, y las grandes preguntas eternas siguen sin respuesta. Élcree que al final, y muy pronto, ambos asuntos encontrarán la solución, pero tiene desconfianza y temores, y estos precisamente residen en el hombre mismo, y no en sus productos científicos. Bajo ese pensamiento murió.

Como científico ya se ha dicho todo. Como filósofo, que lo fue y en gran medida, siempre fue crítico de la corriente tradicional. Vio a la filosofía de otra manera, tal y como tantos otros filósofos-científicos actuales de la talla de Penrose, de Heisenberg, de Schrodinger, de Prigogine. Fue un conocedor de la historia de la filosofía, la que estudió mucho, tanto como criticó sus caminos.

Creo que Hawking reconoció que no hay hoy por hoy respuestas a las grandes preguntas. No es su culpa. Mientras la ciencia no entre en el conocimiento de lo que sucedió antes de los tiempos, de los espacios y de las masas de Planck, no habrá respuesta sobre el universo; mientras no se logre descifrar el misterio del primer coacervado, no habrá respuesta para la vida; mientras no se encuentre el sentido último del hombre en la vida, no habrá respuesta para la humanidad. 
Pero el universo es un enigma. Stephen Hawking intentó descifrarlo, no lo logró, aunque siempre creyó que alguien más lo lograría, y ese alguien tendría que apoyarse, sin duda para él, en los brazos de la ciencia. (pp.144-160)

Reflexión del autor

\section{Referentes bibliográficos}

Hawking, S. (2018). ¿Cómo empezó todo? Breves respuestas a las grandes preguntas (p. 68) [versión de Adobe Acrobat Reader]. Recuperado de http://www.librosmaravillosos.com/breves_respuestas_a_las_grandes_ preguntas/pdf/Breves_resp

Hawking, S. (2018). ¿Cómo empezó todo? Breves respuestas a las grandes preguntas (p. 142) [versión de Adobe Acrobat Reader]. Recuperado de http://www.librosmaravillosos.com/breves_respuestas_a_las_grandes_ preguntas/pdf/Breves_resp

Hawking, S. (2018). ¿Es posible viajar en el tiempo? Breves respuestas a las grandes preguntas (pp. 142-143) [versión de Adobe Acrobat Reader]. Recuperado de http://www.librosmaravillosos.com/breves respuestas_a_las_grandes_preguntas/pdf/Breves_resp

Hawking, S. (2018). ¿Hay un dios? Breves respuestas a las grandes preguntas (p. 52) [versión de Adobe Acrobat Reader]. Recuperado de http://www. librosmaravillosos.com/breves_respuestas_a_las_grandes_preguntas/ pdf/Breves_resp

Hawking, S. (2018). ¿Sobreviviremos en la tierra? Breves respuestas a las grandes preguntas (pp. 144-160) [versión de Adobe Acrobat Reader]. Recuperado de http://www.librosmaravillosos.com/breves respuestas_a_las_grandes_preguntas/pdf/Breves_resp

Hawking, S. (2018). Breves respuestas a las grandes preguntas [versión de Adobe Acrobat Reader]. Recuperado de http://www.librosmaravillosos. com/breves_respuestas_a_las_grandes_preguntas/pdf/Breves_resp

Sanz Lastra, S. (2018). Frases de Stephen Hawking para reflexionar. Recuperado de https://es.linkedin.com/pulse/frases-de-stephen-hawking-parareflexionar-santiago-sanz-lastra-axa- 
Pieza de Exposición

Museo Universitario de Antropología

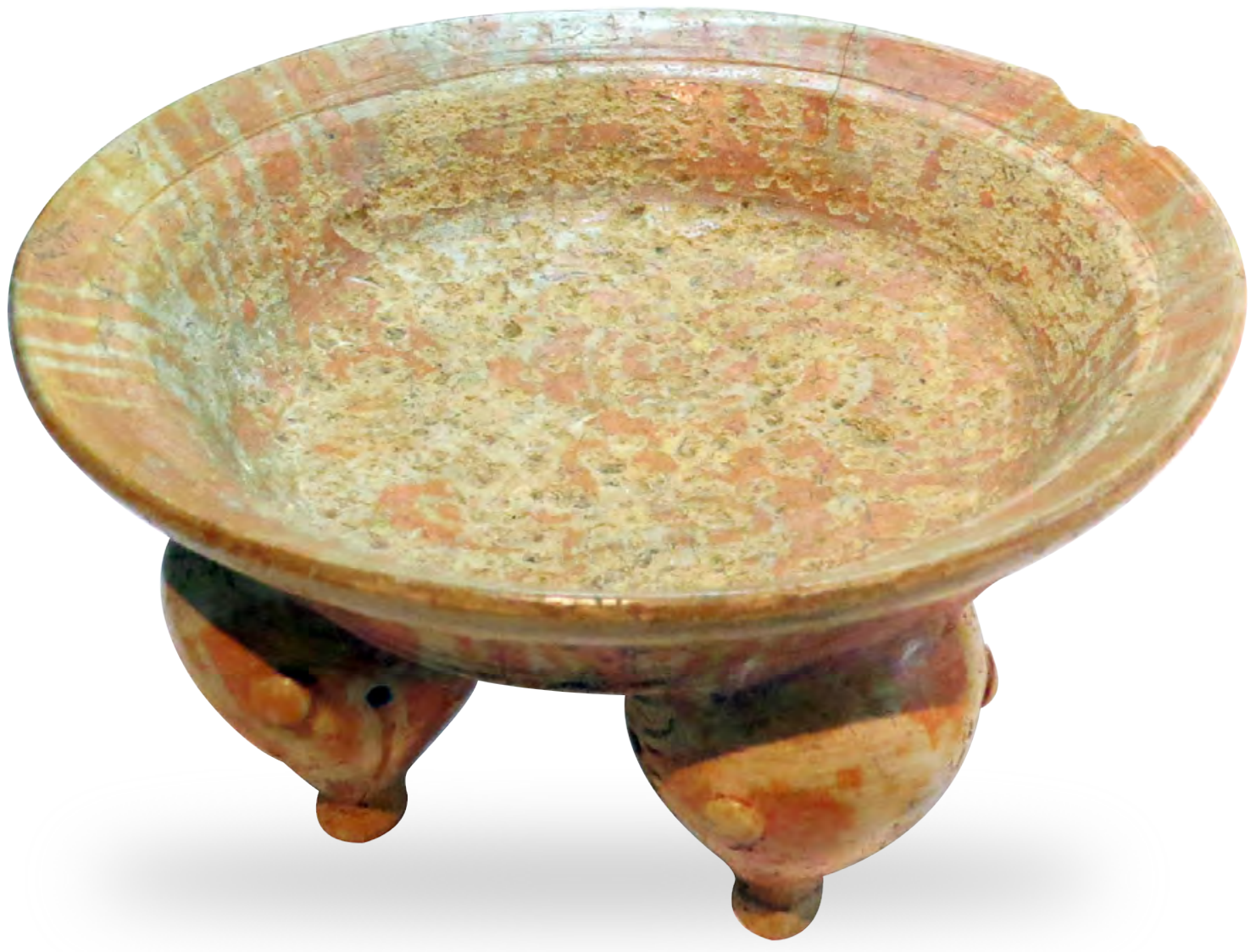

Cuenco

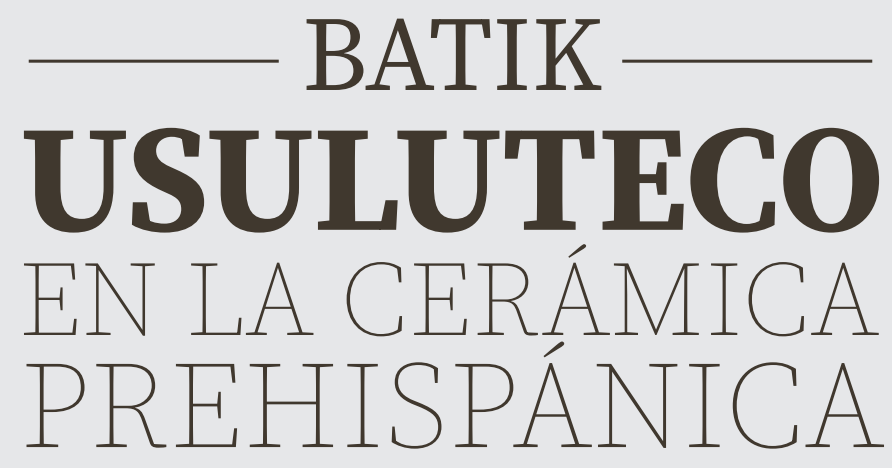

